ISSN 0103-9954

\title{
DEMANDA DOS ESTADOS UNIDOS POR CARVÃO VEGETAL BRASILEIRO
}

\author{
THE DEMAND BY THE UNITED STATES OF AMERICA FOR THE BRAZILIAN CHARCOAL
}

\author{
Jaqueline de Paula Heimann ${ }^{1}$ Andre Ramos Dresch ${ }^{2}$ Alexandre Nascimento Almeida ${ }^{3}$
}

\section{RESUMO}

O objetivo do trabalho é identificar quais variáveis afetam a demanda dos Estados Unidos da América pelo carvão vegetal brasileiro e estimar as elasticidades das mesmas. O estudo utiliza o método dos mínimos quadrados ordinários (MQO), optando pela forma funcional logarítmica, pela simplicidade em obter as elasticidades, e adota o nível de significância de 5\%. Os dados foram obtidos junto ao FAS e ao BEA em período trimestral e corrigidos pelo IPC dos EUA. O modelo ajustado, embora tenha apresentado um baixo coeficiente de determinação $\left(\mathrm{R}^{2}\right)$, não apresentou maiores problemas estatísticos e econométricos. Os resultados indicaram que a demanda dos EUA por carvão brasileiro pode ser explicada pelas expectativas dos consumidores, preço do carvão brasileiro e pela renda norte-americana. Os preços de bens relacionados do México e da Argentina não foram significativos a 5\% de probabilidade, indicando que o carvão desses países não são bons substitutos ao produto brasileiro.

Palavras-chave: carvão vegetal; econometria; comércio internacional.

\begin{abstract}
The objective is to identify which variables affect the demand of the United States of America for the Brazilian charcoal and estimate their elasticity. The study uses the method of ordinary least squares (OLS), opting for the logarithmic functional form because of the simplicity in obtaining elasticity, and adopts the significance level of 5\%. The data were obtained from the FAS and the BEA in quarterly period and deflated by U.S. CPI. The adjusted model, although having a low coefficient of determination $\left(\mathrm{R}^{2}\right)$, did not have greater statistical and econometric problems. The results indicated that the U.S. demand for the Brazilian charcoal can be explained by the expectations of consumers, charcoal price and the U.S. income. The prices of related goods from Mexico and Argentina were not significant at 5\% level, indicating that the charcoal in these countries is not good a substitute for the Brazilian product.
\end{abstract}

Keywords: charcoal; econometrics; international trade.

\section{INTRODUÇÃO}

A cobertura florestal do Brasil, em conjunto com as excelentes condições edafoclimáticas para a silvicultura, conferem ao país inúmeras vantagens comparativas e competitivas para a atividade florestal. Especialistas do setor concordam que há enorme relevância social, econômica e ambiental do setor florestal e sua importância para o desenvolvimento sustentável do país (CARVALHO et al., 2006).

O Brasil possui uma área total de 851 milhões de hectares, sendo 516 milhões de áreas de florestas, das quais 509,8 milhões são florestas naturais e apenas 6,8 milhões de hectares são

1 Engenheira Florestal, M.Sc., Pesquisadora no Núcleo de Estudos e Mediações de Conflitos Ambientais, Rua dos Funcionários, 1540, Juvevê, CEP 80035-050, Curitiba (PR), Brasil. jaquelineheimann@yahoo.com.br

2 Engenheiro Florestal, M.Sc., Universidade Federal do Paraná, Av. Pref. Lothário Meissner, 900, Jardim Botânico, CEP 80210-170, Curitiba (PR), Brasil.

3 Engenheiro Florestal, Dr., professor dos Cursos de Gestão Ambiental e do Agronegócio, Faculdade de Planaltina, Universidade de Brasília, Área Universitária, 1, Vila Nossa Senhora de Fátima, CEP 73300-000, Planaltina (DF), Brasil.

Recebido para publicação em 12/08/2011 e aceito em 23/08/2013 
florestas plantadas, principalmente, com eucalipto e pinus, que juntos representam $93 \%$ do total de plantios, respectivamente, sendo de $66,58 \%$ e de $26,46 \%$.

O setor florestal exerce uma importância considerável na economia brasileira, segundo Pinheiro (2010), a riqueza gerada pelo setor correspondeu $5 \%$ do PIB brasileiro. De acordo com ABRAF (2010), estima-se que o total de empregos (primário e processamento industrial) no segmento de florestas plantadas, em 2009, tenha atingido 3,9 milhões incluindo os diretos (535 mil), indiretos (1,26 milhão) e empregos resultantes do efeito renda (2,16 milhões). O setor ainda contribui significativamente nas exportações totais do Brasil que em 2009 chegaram a US\$ 153,0 bilhões (ABRAF, 2010). Dados do Serviço Florestal Brasileiro (SFB) em 2010 apontam 7,2 bilhões de dólares em exportações do setor florestal brasileiro em 2009, dos quais, US\$ 1,26 bilhão referentes às importações de produtos madeireiros do Brasil pelos Estados Unidos da América.

Segundo a Associação Mineira de Silvicultura (AMA), em 2009, o Brasil teve destaque mundial como maior produtor e/ou mesmo tempo consumidor de carvão vegetal, sendo o único país no mundo em que este insumo tem uma aplicação industrial em larga escala, destinando-se principalmente para a produção de ferro-gusa e aço e ainda ferro-liga e silício metálico.

De acordo com o relatório anual da Associação Brasileira de Produtores de Florestas plantadas (ABRAF), em 2010, o Brasil exporta carvão vegetal para doze países. No ano de 2009 , esses países chegaram a importar 6,4 milhões de toneladas de carvão vegetal, equivalente a US\$ 2,2 milhões. Os Estados Unidos da América é um país detentor de uma das maiores economias do mundo e responsável por $25 \%$ do total de exportações brasileiras, o que equivale a 1,6 milhão de tonelada de carvão vegetal.

Soares et al. (2004) ressaltam a carência de estudos referentes à demanda de carvão vegetal, sugerindo atualizar pesquisar nesta área. Alguns estudos quantitativos relacionados ao segmento de carvão vegetal no Brasil são: Soares et al. (2004), Silva e Silva (1996); Amâncio et al. (1983) e Pereira et al. (1982), porém, não foi encontrado nenhum trabalho quantitativo referente ao comércio internacional de carvão. Apresentada a importância do setor florestal brasileiro, com especial enfoque ao ramo produtor de carvão vegetal, este artigo apresenta os seguintes questionamentos de pesquisa: Que variáveis afetam a demanda dos Estados Unidos por carvão vegetal proveniente do Brasil? $\mathrm{E}$ de que forma o Brasil poderia ser mais presente $\mathrm{e}$ competitivo neste segmento do setor florestal?

O presente trabalho tem como objetivo geral estudar as relações existentes entre o Brasil e seu maior importador de carvão vegetal, os Estados Unidos, bem como analisar as variáveis que influenciam na demanda desse país pelo carvão vegetal brasileiro. Para isso, têm-se como objetivos específicos: entender a relação existente entre o preço do carvão vegetal na conquista de espaço no mercado norte-americano; analisar a relação existente entre Brasil e dois outros países expoentes na exportação de carvão vegetal para o mercado dos Estados Unidos, o México e a Argentina; e compreender a relação das expectativas dos consumidores norte-americanos pelo carvão vegetal brasileiro.

\section{METODOLOGIA}

\section{Referencial teórico}

Do ponto de vista metodológico, o trabalho é caracterizado como de enfoque quantitativo, de caráter explicativo e correlacional. Segundo Sampieri, Collado e Lucio (2006), o enfoque quantitativo busca formular questões de pesquisa e busca respondê-las através de análises estatísticas, pois confia em medições numéricas, enquanto que o enfoque qualitativo baseia-se em um esquema indutivo, seu método de análise é contextual. Para os autores, o caráter explicativo destina-se a estudos que estão destinados a responder as causas dos acontecimentos ou fenômenos, busca responder por que o fenômeno ocorre e em que condições, ou ainda, por que duas ou mais variáveis estão relacionadas, já o caráter correlacional busca avaliar a relação existente entre dois ou mais conceitos, categorias ou variáveis dentro de um determinado contexto.

Gujarati (2000) relata que ao fazer-se uma análise de regressão, busca-se estudar a dependência de uma variável (variável dependente), em relação a uma ou mais variáveis (variáveis explicativas), com intenção de encontrar a média ou o valor médio da dependente em termo de valores conhecidos. Neste sentido, a econometria torna possível fazer uma conjuntura da teoria econômica com medidas concretas. A Econometria dá conteúdo empírico à 
teoria econométrica, ou seja, a teoria econômica postula uma relação entre as variáveis preço e quantidade demandada sem fornecer qualquer dado numérico, cabe à econometria fornecer tais estimativas numéricas (GUJARATI, 2000).

Gujarati (2000) apresenta o caminho que a metodologia econométrica tradicional segue, são elas: (1) formulação da teoria; (2) especificação do modelo matemático; (3) especificação do modelo econométrico; (4) obtenção de dados; (5) estimativa dos parâmetros do modelo econométrico; (6) teste da teoria; (7) previsão ou predição, e (8) utilização do modelo para fins de controle ou política.

Koutsoyiannis (1978) apresenta estas fases resumidas em apenas quatro etapas: (1) formulação da hipótese sustentada; (2) estimação; (3) avaliação do modelo, e (4) avaliação do poder de previsão do modelo.

Este trabalho se baseia nas metodologias propostas por Gujarati (2000) e Koutsoyiannis (1978), no entanto, apoia-se nos passos propostos por este último autor. Não é objetivo deste estudo, utilizar os resultados da análise econométrica para prever valores futuros das variáveis dependentes e independentes, logo, não será feita previsão ou predição do modelo.

Os gostos e preferências serão desconsiderados por falta de dados. Desta forma, o modelo econométrico explicativo da demanda dos Estados Unidos pelo carvão vegetal brasileiro é demonstrado na equação 1 .

$$
\begin{aligned}
\ln D M_{t}^{E U A A}= & \alpha_{1}+\alpha_{2} \ln P_{t}^{B R A}+\alpha_{3} \ln R_{t}+\alpha_{4} \ln P B R_{t}^{M E X}+ \\
& \alpha_{5} \ln P B R_{t}^{A R G}+\alpha_{6} \ln \operatorname{Exp}_{t-1}^{E U A}+\varepsilon_{t}
\end{aligned}
$$

Em que: = Demanda dos Estados Unidos por carvão vegetal brasileiro; $P_{t}^{B R A}=$ Preço pago pelos Estados Unidos pelo carvão vegetal do Brasil; $R_{t}=$ Renda dos Estados Unidos; $P B R_{t}^{M E X}=$ Preço do bem relacionado mexicano; $P B R_{t}^{A R G}=$ Preço do bem relacionado argentino; $\operatorname{Exp}_{t-1}^{E U A}=$ Expectativas dos consumidores dos Estados Unidos; $\varepsilon_{t}=$ Termo de erro da equação.

Existem outras variáveis que afetam a demanda dos EUA por carvão vegetal como, por exemplo: a taxa de juros, a distribuição de renda, outros potenciais concorrentes como a Holanda e o
Canadá, os gostos e preferências dos consumidores (desconsiderados deste modelo), as tarifas de importação, os acordos comerciais entre Estados Unidos e países exportadores do produto, entre outras (ALMEIDA et al., 2010). Porém, problemas de multicolinearidade, dificuldades para obter os dados na periodicidade desejada, simplicidade do modelo e a percepção pelos autores de menor importância de algumas variáveis, conduziram à omissão dessas variáveis na função.

Segundo Gujarati (2000), a influência de fatores menos importantes é considerada pela introdução no modelo de uma variável aleatória, que pode ser expressa pelo termo de erro da equação $\left(\varepsilon_{t}\right)$.

De acordo com Almeida et al. (2007), as expectativas dos consumidores sobre os níveis futuros de preço, os níveis futuros de rendimento e a disponibilidade futura de bens têm a possibilidade de influenciar o comportamento corrente das compras. Se os consumidores acreditam que os preços dos bens irão subir no futuro, haverá um forte incentivo para antecipar suas compras e, assim, evitar o pagamento com preços mais altos. $\mathrm{O}$ autor apresentou um modelo de oferta de madeira em tora para processamento mecânico no estado do Paraná em que capta a influência das expectativas dos preços futuros através de valores passados da série de preço.

As hipóteses admitidas no trabalho são fundamentadas conforme a lei de demanda, estando demonstradas na Tabela 1. Para a análise estatística foram considerados significativos aqueles valores com um nível de até 5\%.

Quanto à demanda dos EUA pelo carvão vegetal brasileiro é esperada uma relação negativa com o preço do produto brasileiro e de bens complementares, e positiva com a expectativa, renda e preço de bens substitutos (MANKIW, 2001). Segundo Pindyck e Rubinfeld (2002), quando a elasticidade de um variável for um número superior a 1, a demanda é elástica a variável; se a elasticidade for menor que $1 \mathrm{em}$ magnitude, a demanda é inelástica.

\section{Referencial analítico}

Para a estimativa da equação 1 utilizou-se o método dos Mínimos Quadrados Ordinários (MQO) optando-se pela forma funcional logarítmica devido à simplicidade de se obter as elasticidades 
TABELA 1: Hipóteses referentes aos parâmetros.

TABLE 1: Hypotheses regarding to the parameters.

\begin{tabular}{cccc}
\hline Coeficiente & Variáveis e constantes & Sinal esperado & $\begin{array}{c}\text { Nível de } \\
\text { confiança } \\
\text { esperado }\end{array}$ \\
\hline$\alpha_{1}$ & $\mathrm{Cte}^{1}$ & - & \\
$\alpha_{2}$ & $P_{t}^{B R A}$ & $<0$ & \\
$\alpha_{3}$ & $R_{t}$ & $>0$ & \\
$\alpha_{4}$ & $P B R_{t}^{M E X}$ & $>0$ & \\
$\alpha_{5}$ & $P B R_{t}^{A R G}$ & $>0$ & \\
$\alpha_{6}$ & $\operatorname{Exp}_{t-1}^{E U A}$ & & \\
\hline
\end{tabular}

Em que: ${ }^{1}$ Constante.

diretamente (ALMEIDA et al., 2007; ALMEIDA et al., 2010). Segundo Gujarati (2000), o MQO tem propriedades estatísticas bastante significativas, o que o torna um método poderoso e popular de análise de regressão.

Para obter o preço pago pelo carvão vegetal, fez-se a razão entre o valor pago pelos EUA e quantidade importada por este país de cada um dos fornecedores analisados (Brasil, México e Argentina). Os dados fornecidos pelo FAS - Foreign Agricultural Service (2011), não discriminam as espécies das quais são produzidas o carvão vegetal exportado para os EUA. Conforme Angelo et al. (2009), em trabalhos quantitativos é bastante comum que índices de quantidade e preço que agregam diversos bens sejam utilizados, o que a princípio, não tem nenhum problema, porém, é importante que sejam respeitados os objetivos propostos pelo estudo e a avaliação dos resultados seja cautelosa.

$\mathrm{O}$ presente estudo verificou as hipóteses referentes à ausência de problemas de multicolinearidade, heteroscedasticidade, autocorrelação e especificação por meio de procedimentos amplamente difundidos na literatura.

Para o teste de multicolinearidade foi analisado o $\mathrm{R}^{2}$, as razões $t$, bem como o indicador do Fator de Inflação de Variância (FIV). O Teste Geary (1970), também conhecido como Teste das Carreiras, foi aplicado para autocorrelação. Usualmente é utilizado o teste tradicional estatística d de Durbin-Watson, porém, segundo Almeida et al. (2010) e Angelo et al. (2009), este teste não se aplica em modelos que apresentam componentes autorregressivos. O teste utilizado para verificar a ausência de heteroscedasticidade e especificação foi o Teste de White (1980). O Teste de White é de fácil aplicação e ainda possibilita a avaliação dos dois problemas em conjunto. Além dos testes de carreiras e White, analisou-se a dispersão de resíduos como um primeiro diagnóstico informal para detectar autocorrelação, heteroscedasticidade ou especificação (GUJARATI, 2000).

\section{Banco de dados}

Os dados referentes a valores e quantidades importados de carvão vegetal pelos Estados Unidos da América originários do Brasil, Argentina e México, foram coletados junto ao Foreign Agricultural Service - FAS (2011), já a renda norteamericana foi obtida junto ao Bureau of Economic Analysis - BEA (2010).

Os dados coletados possuem periodicidades trimestrais (quatro séries por ano) no período compreendido entre os anos de 2000 e 2010, e foram corrigidos através do Índice de Preços ao Consumidor nos Estados Unidos - IPC, tomando como base o último trimestre do ano de 2010. Os dados utilizados são apresentados na Tabela 2.

\section{RESULTADOS E DISCUSSÕES}

O modelo ajustado da demanda dos 
TABELA 2: Banco de dados utilizados na análise, em valores nominais, período de 2000 a 2010.

TABLE 2: Analysis database in nominal terms, period from 2000 to 2010.

\begin{tabular}{|c|c|c|c|c|c|c|c|}
\hline Ano & Período & $\begin{array}{c}\mathrm{P}_{\mathrm{t}}^{\mathrm{BRA}}(\mathrm{USS} \$ \\
\mathrm{mil} / \mathrm{t})\end{array}$ & $\begin{array}{c}\text { PBR }_{\mathrm{t}}^{\mathrm{MEX}} \\
\text { (US\$ mil/t) }\end{array}$ & $\begin{array}{c}\text { PBR }_{\mathrm{t}}^{\text {ARG }} \\
\text { (US\$ mil/t) }\end{array}$ & $\mathrm{R}_{\mathrm{t}}$ (US\$ bilhões) & $\mathrm{DM}_{t}^{\mathrm{EUA}}(\mathrm{t})$ & $\operatorname{EXP}_{t-1}^{\text {EUA }}(t)$ \\
\hline \multirow[t]{4}{*}{2000} & $\mathrm{~T} 1$ & 0,27 & 0,25 & 0,41 & 12510 & 179 & 390 \\
\hline & $\mathrm{T} 2$ & 0,19 & 0,24 & 0,43 & 12684 & 261 & 179 \\
\hline & $\mathrm{T} 3$ & 0,30 & 0,26 & 0,35 & 12668 & 143 & 261 \\
\hline & $\mathrm{T} 4$ & 0,31 & 0,33 & 0,44 & 12744 & 92 & 143 \\
\hline \multirow[t]{4}{*}{2001} & $\mathrm{~T} 1$ & 0,31 & 0,31 & 0,37 & 12664 & 92 & 92 \\
\hline & $\mathrm{T} 2$ & 0,31 & 0,28 & 0,44 & 12704 & 203 & 92 \\
\hline & $\mathrm{T} 3$ & 0,31 & 0,29 & 0,37 & 12687 & 190 & 203 \\
\hline & $\mathrm{T} 4$ & 0,24 & 0,23 & 0,46 & 12807 & 315 & 190 \\
\hline \multirow[t]{4}{*}{2002} & $\mathrm{~T} 1$ & 0,29 & 0,26 & 0,55 & 12918 & 240 & 315 \\
\hline & $\mathrm{T} 2$ & 0,31 & 0,25 & 0,25 & 12907 & 193 & 240 \\
\hline & $\mathrm{T} 3$ & 0,28 & 0,24 & 0,32 & 12971 & 270 & 193 \\
\hline & $\mathrm{T} 4$ & 0,32 & 0,22 & 0,23 & 13007 & 122 & 270 \\
\hline \multirow[t]{4}{*}{2003} & $\mathrm{~T} 1$ & 0,30 & 0,23 & 0,26 & 13032 & 248 & 122 \\
\hline & $\mathrm{T} 2$ & 0,38 & 0,25 & 0,28 & 13117 & 319 & 248 \\
\hline & $\mathrm{T} 3$ & 0,31 & 0,23 & 0,30 & 13347 & 243 & 319 \\
\hline & $\mathrm{T} 4$ & 0,28 & 0,27 & 0,30 & 13538 & 403 & 243 \\
\hline \multirow[t]{4}{*}{2004} & $\mathrm{~T} 1$ & 0,29 & 0,24 & 0,28 & 13627 & 1000 & 403 \\
\hline & T2 & 0,19 & 0,24 & 0,29 & 13649 & 3059 & 1000 \\
\hline & $\mathrm{T} 3$ & 0,35 & 0,24 & 0,29 & 13797 & 2614 & 3059 \\
\hline & $\mathrm{T} 4$ & 0,34 & 0,21 & 0,25 & 13941 & 1668 & 2614 \\
\hline \multirow[t]{4}{*}{2005} & $\mathrm{~T} 1$ & 0,34 & 0,27 & 0,26 & 14121 & 950 & 1668 \\
\hline & $\mathrm{T} 2$ & 0,26 & 0,30 & 0,27 & 14087 & 4240 & 950 \\
\hline & $\mathrm{T} 3$ & 0,20 & 0,28 & 0,28 & 14165 & 1802 & 4240 \\
\hline & $\mathrm{T} 4$ & 0,39 & 0,23 & 0,29 & 14286 & 396 & 1802 \\
\hline \multirow[t]{4}{*}{2006} & $\mathrm{~T} 1$ & 0,32 & 0,28 & 0,28 & 14509 & 96 & 396 \\
\hline & $\mathrm{T} 2$ & 0,26 & 0,30 & 0,25 & 14443 & 300 & 96 \\
\hline & $\mathrm{T} 3$ & 0,33 & 0,30 & 0,27 & 14478 & 435 & 300 \\
\hline & $\mathrm{T} 4$ & 0,38 & 0,38 & 0,27 & 14772 & 152 & 435 \\
\hline \multirow[t]{4}{*}{2007} & $\mathrm{~T} 1$ & 0,29 & 0,28 & 0,24 & 14818 & 203 & 152 \\
\hline & $\mathrm{T} 2$ & 0,35 & 0,29 & 0,31 & 14764 & 337 & 203 \\
\hline & $\mathrm{T} 3$ & 0,46 & 0,29 & 0,30 & 14886 & 522 & 337 \\
\hline & $\mathrm{T} 4$ & 0,53 & 0,36 & 0,31 & 14918 & 148 & 522 \\
\hline \multirow[t]{4}{*}{2008} & $\mathrm{~T} 1$ & 0,47 & 0,36 & 0,36 & 14788 & 154 & 148 \\
\hline & $\mathrm{T} 2$ & 0,57 & 0,28 & 0,39 & 14612 & 238 & 154 \\
\hline & $\mathrm{T} 3$ & 0,56 & 0,25 & 0,44 & 14458 & 170 & 238 \\
\hline & $\mathrm{T} 4$ & 0,49 & 0,23 & 0,46 & 14577 & 259 & 170 \\
\hline \multirow[t]{4}{*}{2009} & $\mathrm{~T} 1$ & 0,32 & 0,28 & 0,49 & 14507 & 495 & 259 \\
\hline & $\mathrm{T} 2$ & 0,55 & 0,29 & 0,33 & 14336 & 465 & 495 \\
\hline & $\mathrm{T} 3$ & 0,61 & 0,28 & 0,40 & 14324 & 465 & 465 \\
\hline & $\mathrm{T} 4$ & 0,60 & 0,28 & 0,42 & 14462 & 143 & 465 \\
\hline
\end{tabular}

Continua ... 
TABELA 2: Continuação...

TABLE 2: Continued...

\begin{tabular}{|c|c|c|c|c|c|c|c|}
\hline Ano & Período & $\begin{array}{c}\mathrm{P}_{\mathrm{t}}^{\mathrm{BRA}}(\mathrm{USS} \$ \\
\mathrm{mil} / \mathrm{t})\end{array}$ & $\begin{array}{c}\mathrm{PBR}_{\mathrm{t}}^{\mathrm{MEX}} \\
\text { (US\$ mil/t) }\end{array}$ & $\begin{array}{c}\mathrm{PBR}_{\mathrm{t}}^{\text {ARG }} \\
(\mathrm{USS} \mathrm{mil} / \mathrm{t})\end{array}$ & $\mathrm{R}_{\mathrm{t}}$ (US\$bilhões) & $\mathrm{DM}_{\mathrm{t}}^{\mathrm{EUA}}(\mathrm{t})$ & $\operatorname{EXP}_{t-1}{ }^{E U A}(t)$ \\
\hline \multirow[t]{4}{*}{2010} & $\mathrm{~T} 1$ & 0,60 & 0,32 & 0,37 & 14573 & 244 & 143 \\
\hline & $\mathrm{T} 2$ & 0,66 & 0,34 & 0,35 & 14632 & 285 & 244 \\
\hline & $\mathrm{T} 3$ & 0,55 & 0,32 & 0,40 & 14786 & 183 & 285 \\
\hline & $\mathrm{T} 4$ & 0,58 & 0,31 & 0,40 & 14861 & 76 & 183 \\
\hline
\end{tabular}

Fonte: FAS (2010) e BEA (2010).

Estados Unidos por carvão vegetal brasileiro está representado na equação 2 e os valores encontrados para o coeficiente de determinação $\left(\mathrm{R}^{2}\right)$ e para o teste de F são apresentados a seguir.

$$
\begin{aligned}
\ln D M_{t}^{E U A A}= & \alpha_{1}+\alpha_{2} \ln P_{t}^{B R A}+\alpha_{3} \ln R_{t}+\alpha_{4} \ln P B R_{t}^{M E X}+ \\
& \alpha_{5} \ln P B R_{t}^{A R G}+\alpha_{6} \ln \operatorname{Exp}_{t-1}^{E U A}+\varepsilon_{t}
\end{aligned}
$$

Em que: $\mathrm{R}^{2} \mathrm{aj}=0,54 ; \mathrm{F}=11,09$.

O coeficiente de determinação ajustado $\left(\mathrm{R}^{2} \mathrm{aj}\right)$ indica que $54 \%$ da variância da demanda de carvão vegetal brasileiro pelos Estados Unidos é explicado pelo modelo. De acordo com Maroco (2007), o valor do $\mathrm{R}^{2}$ que se espera produzir de um ajustamento, é um valor subjetivo. $\mathrm{O}$ autor ressalta que, enquanto para ciências exatas os valores geralmente aceitos são $\mathrm{R}^{2}>0,9$, para as ciências sociais valores de $\mathrm{R}^{2}>0,5$ consideram já aceitável o ajustamento. Além disso, seguindo os conselhos de Gujarati (2000) e Goldberger (1991), minimizouse a importância do $\mathrm{R}^{2} \mathrm{aj}$, pois o objetivo do estudo não é obter um $\mathrm{R}^{2}$ alto, mas sim obter estimativas confiáveis dos verdadeiros coeficientes de regressão da população e fazer inferências estatísticas sobre eles. Ainda conforme Goldberger (1991), um R ${ }^{2}$ alto não é nenhuma evidência em favor do modelo e um baixo $\mathrm{R}^{2}$ não é nenhuma evidência contra ele.

$\mathrm{O}$ teste $\mathrm{F}$ avalia a hipótese de que todos os coeficientes são simultaneamente iguais a zero, esta hipótese foi rejeitada a $5 \%$ de probabilidade.

A Tabela 3 compila os resultados da regressão, razões de $t$, e os fatores de inflação de variância, assim como os respectivos níveis de significância.

O modelo apresentou, em todas as variáveis, a direção de respectivos sinais que se esperava através das hipóteses formuladas. Porém, os Preços de bens relacionados ( $P B R_{t}^{M E X}$ e $P B R_{t}^{A R G}$ ) não foram significativos ao nível de $5 \%$ de probabilidade.

Observando os dados resultantes da análise, é possível afirmar que, aparentemente, o modelo não apresentou problemas de multicolinearidade. De acordo com Gujarati (2000), há uma regra prática para diagnosticar a multicolinearidade, quando se observa um $\mathrm{R}^{2}$ alto e poucas razões $t$ significativas, então há multicolinearidade. No entanto, isto não ocorreu neste modelo. Maroco (2007) destaca outra forma de identificar a multicolinearidade através dos valores de Fator de Inflação da Variância (FIV), de acordo com este autor, valores de FIV superiores a 5 ou mesmo 10, apresentam problemas de multicolinearidade.

$\mathrm{O}$ modelo ajustado não apresentou problemas de autocorrelação, heteroscedasticidade e especificação como sugerido pela dispersão aleatória dos resíduos (Figura 1). Esta afirmação é confirmada por meio da análise dos testes Geary (1970) e White (1980), que rejeitaram, a 5\% de significância, a presença de problemas de autocorrelação, heteroscedasticidade e especificação, o que não foi o caso.

Os resultados indicaram um coeficiente de elasticidade unitária para o preço do carvão vegetal brasileiro, altamente elástico à renda total americana, elasticidade unitária para o preço do bem relacionado do México, e inelástico para preço do bem relacionado da Argentina e para as expectativas. No entanto, os preços dos bens substitutos, neste modelo, não foram estatisticamente significativos ao nível de 5\% de significância.

Embora o carvão, independente de onde é produzido, é semelhante, isso não garante que sejam bons substitutos no mercado, pois a concorrência entre os produtos depende, além de características físicas, de: garantia de fornecimento, costumes, arranjos políticos, institucionais, creditícios entre 
TABELA 3: Resultados obtidos pelo ajustamento do modelo.

TABLE 3: Results obtained by adjusting the model.

\begin{tabular}{ccccc}
\hline Variável & coeficiente & $T$ & Sig. & FIV \\
\hline Constante & $-44,789$ & $-1,86$ & 0,71 & \\
\hline$P_{t}^{B R A}$ & $-1,038$ & $-2,50$ & 0,00 & 1,95 \\
$P B R_{t}^{M E X}$ & $-1,066$ & $-1,23$ & 0,11 & 1,53 \\
$P B R_{t}^{A R G}$ & 0,039 & 0,08 & 0,47 & 1,34 \\
$R_{t}$ & 4,690 & 1,88 & 0,03 & 2,33 \\
$\operatorname{Exp}_{t-1}^{E E A}$ & 0,589 & 4,75 & 0,00 & 1,36 \\
\hline
\end{tabular}

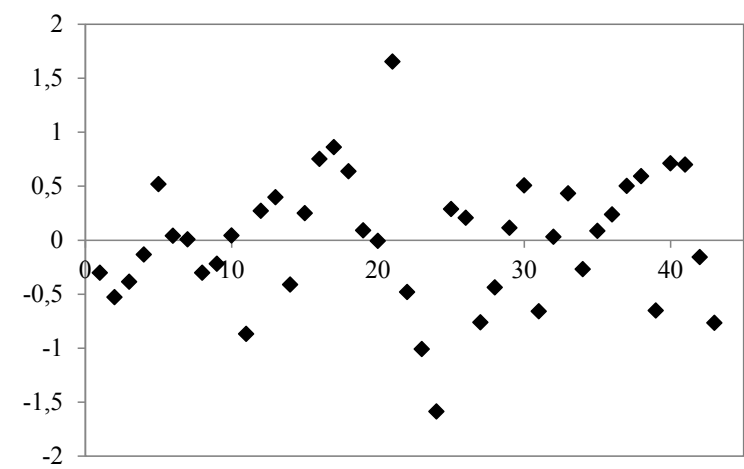

FIGURA 1: Dispersão dos resíduos do modelo. FIGURE 1: Residual dispersion of the model.

outros (FONTES e BARBOSA, 1991 e ALMEIDA et al., 2010).

O resultado encontrado para a elasticidade preço do carvão vegetal brasileiro demonstra que se trata de um produto com elasticidade unitária, desta forma, uma alteração em $1 \%$ no preço do carvão vegetal brasileiro acarretaria uma alteração em $1 \%$ da demanda, em sentido contrário, quando analisado isoladamente, ou seja, ceteris paribus. Resultado contrário ao encontrado por Soares et al. (2004) que, analisando o mercado de carvão vegetal brasileiro no período de 1974 a 2000, concluíram que uma variação de $10 \%$ no preço do produto ocasionaria uma redução de $1 \%$ na quantidade demandada, indicando que a demanda por carvão vegetal brasileiro é inelástica em relação ao preço. É importante ressaltar que a análise de Soares et al. (2004) remete ao mercado interno, logo a inelasticidade encontrada é aceitável, visto que o carvão vegetal é fundamental para o funcionamento da indústria siderúrgica. No entanto, ao se estudar as exportações brasileiras para o mercado externo, espera-se que o país importador busque alternativas ao se deparar com um aumento no preço do produto. Sendo assim, é provável que, ao encontrar preços mais elevados do carvão vegetal brasileiro, a indústria dos Estados Unidos procure este mesmo insumo em outros países, como a China, ou até mesmo substitua-lo pelo carvão mineral.

Foi possível verificar através deste estudo que a renda dos Estados Unidos foi a variável de maior influência nas importações de carvão vegetal produzido no Brasil. A ordem de grandeza indica que se a renda dos EUA (PIB) cair em um ponto de percentagem, a quantidade importada de carvão vegetal brasileiro decresce o quádruplo $(4,69 \%)$. Resultado semelhante ao encontrado por Almeida et al. (2010), no qual os autores concluem que uma queda de $1 \%$ na renda norte-americana levaria a uma queda de $2,07 \%$ da quantidade importada por esse país das molduras brasileiras.

A relação direta no sinal da expectativa do consumidor norte-americano por carvão vegetal brasileiro esteve de acordo com Brännlund et al. (1985), segundo o qual, um ano bom seguido de um aumento da quantidade importada no presente leva a uma expectativa otimista e um aumento das importações no futuro. A ordem de grandeza para as expectativas foi de 0,589 pontos de percentagem, quando a quantidade importada de moldura pelos EUA em um período defasado aumenta $1 \%$. 


\section{CONCLUSÕES E RECOMENDAÇÕES}

A demanda dos Estados Unidos por carvão vegetal brasileiro pode ser explicada pelas expectativas (preço do carvão vegetal defasado em um período), pelo preço pago pelos EUA por carvão vegetal brasileiro e pela renda total dos Estados Unidos.

Os resultados apontam que a renda é a variável de maior influência nas importações do carvão vegetal brasileiro pelos Estados Unidos, demonstrando que uma queda na renda dos Estados Unidos levaria a uma queda mais de quatro vezes maior da importação de carvão vegetal brasileiro. Estes resultados sugerem a existência de uma forte dependência entre a conquista de mercado pelo produto brasileiro e o crescimento dos EUA ou, seja, a capacidade do Brasil conquistar mercado nos Estados Unidos em momentos de baixa expansão econômica através do confronto com a concorrência é baixa.

Haja vista que os Estados Unidos são um dos maiores importadores do carvão vegetal brasileiro, é aconselhável que este segmento do setor florestal, até mesmo em razão da instabilidade econômica americana dos últimos anos, comece a buscar novos mercados, tornando-se menos dependente da economia norte-americana.

A análise do modelo estimado leva à conclusão de que a demanda dos Estados Unidos por carvão vegetal brasileiro não é explicada pelo preço dos bens relacionados do México e Argentina, pois estes não foram significativos a $5 \%$ de probabilidade, sugerindo serem produtos identificados como diferentes pelo consumidor dos EUA.

A elasticidade preço da demanda acima da unitária é um indicativo de que o carvão vegetal brasileiro pode estar enfrentando forte concorrência para ganhar espaço no mercado dos EUA, logo, uma redução de seu preço poderia proporcionar grande vantagem competitiva em curto prazo, porém, a inferência não deve ser extrapolada para médio e longo prazo, cabendo neste caso sugerir que novos estudos sejam realizados a este respeito.

$\mathrm{O}$ efeito das expectativas foi ao encontro da literatura, de forma que um aumento das importações no presente leva a uma expectativa positiva para um aumento futuro. Desta forma, é importante que o Governo Federal Brasileiro, por meio de políticas públicas que contribuam para um câmbio mais competitivo e redução de impostos, incentive a exportação deste produto para o mercado dos Estados Unidos, visando estimular a consolidação desse mercado.

\section{REFERÊNCIAS BIBLIOGRÁFICAS}

ABRAF. Anuário estatístico da Abraf 2010 ano base 2009. - Brasília, 2010. $140 \mathrm{p}$.

ALMEIDA, A. N. et al. Mercado de madeiras tropicais: substituição na demanda de exportação. Acta Amazônica, v. 40, n. 1, p. 119 - 126, 2010.

ALMEIDA, A. N. et al. Análise econométrica do preço pago as exportações de compensados paranaenses . In: ENCONTRO DE ECONOMIA PARANAENSE ECOPAR, 5., 2007, Curitiba. p. 1-70. Disponível em: <http://www.ecopar.ufpr. br/artigos/a7_082.pdf $>$ Acesso em: 21/03/2011.

ALMEIDA, A. N.; SILVA, J. C. G. L.; ANGELO, H. AnáliseeconométricadademandadosEstadosUnidos por moldura de Pinus no Brasil. Scientia Forestalis, Piracicaba, v. 38, n.87. Piracicaba: set. 2010. p.491-498.

AMÂNCIO, M. R. C.; BRANDT, S. A.; PEREIRA, A. R.; Modelo recursivo da oferta e demanda de carvão vegetal para a siderurgia no Estado de Minas Gerais. Brasil Florestal, Brasília, n. 56, p. 31 - 56, 1983.

ASSOCIAÇÃO MINEIRA DE SILVICULTURA - AMS. Números do setor. Belo Horizonte: AMS, 2009. Disponível em: $<$ http://www.silviminas.com. br>. Acesso em: 15 maio 2011.

ANGELO, H.; ALMEIDA, A. N.; SERRANO, A. L. M. Determinantes da demanda de madeira em toras para celulose no Brasil. Scientia Forestalis, Piracicaba, v. 37, n. 84. p. 491-498, dez. 2009.

BEA - Bureau of Economic Analysis. Departament of Commerce of U.S. National Economic Accounts. Produto Interno Bruto (Tabela 1.1.5), 2010. Disponível em: <http://www.bea.gov/national/ nipaweb/TableView.asp?SelectedTable $=5 \&$ ViewS eries $=$ NO $\&$ Java $=$ no $\&$ Request 3 Place $=$ N $\& 3$ Place $=$ N\&FromView $=$ YES\&Freq $=$ Qtr\&FirstYear $=1988$ $\&$ LastYear $=2010 \& 3$ Place $=$ N\&Update $=$ Update $\& \mathrm{Ja}$ vaBox=no\#> Acesso em: 10/03/2011.

BRÄNNLUND, R.; JOHANSSON, P.O; LOFGREN, K.G. An econometric analysis of aggregate sawtimber and pulpwood supply in Sweden. Forest Science, Maryland, v. 31, n. 3, p. 595-606, 1985. 
CARVALHO, R. M. M. A.; SOARES, T. S.; VALVERDE, S. R. Setor florestal é destaque na economia brasielira. Remade, Abril, 2006. Disponível em: <http:// www.remade.com.br/br/artigos_tecnicos. hp sub $=43 \&$ categoria $=\&$ subcategoria $=$ Economia $>$ Acesso em: 02/03/2011.

FONTES, R. M. O.; BARBOSA, M. L. Efeitos da integração econômica do Mercosul e da Europa na competitividade das exportações brasileiras de soja. Revista de Economia e Sociologia Rural, Brasília, v. 29, n. 4, p. 335-351, 1991.

FOREIGN AGRICULTURAL SERVICE - FAS. Disponível em: $<$ http://www.fas.usda.gov/ $>$. Acesso em: 18/03/2011.

GEARY, R. C. Relative efficiency of count of sign changes for assessing residual autoregression in least squares regression. Biometrika, Cambridge, v. 57, p. 123-127, 1970

GOLDBERGER, A. S. A Course in Econometrics. London: Havard University Press, Cambridge, Massachusetts, 1991. 405 p.

GUJARATI, D. N. Econometria básica. 3. ed. São Paulo: MAKRON Books, 2000. 846 p.

WHITE, H. A Heteroscedasticity Consistent Covariance Matrix Estimator and a Direct Test of Heteroscedasticity. Econometrica, v. 48, 1990, p. 817-818.

KOUTSOYIANNIS, A. Theory of Econometrics: an introductory exposition of econometric methods. 2nd ed. New Jersey: Barnes\&Noble Books, 1978. $683 \mathrm{p}$.

MANKIW, N. G. Introdução à Economia: Princípios de Micro e Macroeconomia. 2. ed. Rio de Janeiro: Elsevier, 2001. 831 p.

MAROCO, J. Análise estatística - Com utilização do SPSS. 3. ed. Lisboa: Silabo, 2007. $822 \mathrm{p}$.

OLIVEIRA, A. C. et al. Parâmetros de qualidade da madeira e do carvão vegetal do Eucalyptus pellita $\mathrm{F}$. Muell. Scientia Forestalis, Piracicaba, v. 38, n. 87, p. 431-439, set. 2010.

PEREIRA, A. R.; BRANDT, S. A.; TEXEIRA, H. H. L. - Análise econométrica da demanda de carvão vegetal. Revista Árvore, Viçosa, v. 6, n. 2, p. $99-103,1982$.

PINDYCK, R.; RUBINFELD, D. .L. Microeconomia. 5. ed. São Paulo: Prentice Hall, 2002.

PINHEIRO, G. Florestas no Brasil: Sistema altamente sustentável. Remade. Junho, 2010. Disponível em: <http://www.remade.com.br/ br/noticia.php?num $=7444 \&$ title $=$ Florestas $\% 20$ no $\% 20$ Brasil:\%20Sistema $\% 20$ altamente $\% 20$ sustent $\%$ E1 vel> Acesso em: 04/03/2011.

SFB - Serviço Florestal Brasileiro. Florestas do Brasil em resumo - 2010: dados de 2005-2010. / Serviço Florestal Brasileiro. - Brasília: SFB, 2010. $152 \mathrm{p}$.

SILVA, M. L. e SILVA, J. M. A. - Análise do comportamento temporal dos preços do carvão vegetal: aplicação e avaliação da metodologia "Box and Jenkins". Revista Árvore, Viçosa, v. 20, n. 1, p. $57-67,1996$.

SOARES, N. S.; SILVA, M.; FONTES, A. A. Análise econométrica do mercado brasileiro de carvão vegetal no período de 1974 a 2000. Scientia Forestalis, Piracicaba, n. 66, p. 84-93, dez. 2004.

REMADE. Estados Unidos - Exportações diminuem, mas importância comercial permanece. Revista da Madeira, Curitiba, Artigos Técnicos. Maio de 2008-a.

REMADE. O lado bom do carvão. Revista da Madeira, Curitiba, edição n. 114 - Jun. 2008-b.

REMADE. Banco de dados: Carvão Vegetal. Revista da Madeira. Disponível em: $<$ http://www. remade.com.br/br/bd_carvao_vegetal.php $>$ Acesso em: 04/03/2011.

SAMPIERI, R. H.; COLLADO, C. F.; LUCIO, P. B. Metodologia de pesquisa. 3. ed. São Paulo: McGraw-Hill, 2009. 